\title{
Poster title: Prevalence of aortic valve sclerosis and associated factors among type 2 diabetes mellitus patients in Cuba
}

\author{
Y. Pina Rivera ${ }^{1}$, L. Cruz Benites ${ }^{2}$, G.M. Rwegerera ${ }^{1}$, J.C. Hechevarria Parlay ${ }^{3}$. \\ 1 University of Botswana, Internal Medicine, Gaborone, Botswana. \\ ${ }^{2}$ Dr. Carlos J. Finlay Hospital, Cardiology, Havana, Cuba. \\ ${ }^{3}$ Dr. Carlos J. Finlay Hospital, Internal Medicine, Havana, Cuba
}

\section{Background}

Aortic valve sclerosis (AVS) is associated with an increased risk of all-cause and cardiovascular mortality in Type 2 diabetic patients, independently of established risk factors. ${ }^{1}$ There is limited global data on burden and risk factors for AVS; specifically epidemiology of AVS among diabetes mellitus patients in Cuba is not documented.

\section{Aim}

The aim of this study was to obtain the prevalence of aortic valve sclerosis in patients with type 2 diabetes mellitus and as well determine association between AVS and sociodemographic and biochemical parameters.

\section{Method}

We conducted a cross- sectional study to identify the prevalence of AVS among asymptomatic Type 2 diabetic patients attending Dr. Carlos J. Finlay Hospital in Havana, Cuba from May 2010 to May 2012. A resting M- mode Echocardiography was performed in 79 patients without history of hypertension, heart failure, valvular heart diseases or kidneys diseases.

AVS was defined according to international guidelines as either focal or diffuse calcification of the aortic leaflets without significant obstruction to left ventricular outflow, with a transaortic peak instantaneous velocity $<2.6 \mathrm{~m} / \mathrm{s}$. ${ }^{2}$ A comparison was made between patients with AVS and those without AVS to determine association with sociodemographic and biochemical parameters. A p-value $<0.05$ was considered statistically significant.

\section{Results}

Aortic valve sclerosis was found in 18/79 (22.8\%); and was significantly associated with elderly age $(p<0.001)$, and high level of total cholesterol $(p<0.04)$. Gender, body mass index, fasting blood sugar, post-postprandial glucose, triglycerides, $\mathrm{HbA} 1 \mathrm{c}$ were not statistically associated with AVS.

\section{Discussion}

Aortic valve sclerosis was very common in type 2 diabetic patients without any other apparent cardiovascular disease and it appeared to correlate broadly to the elderly age and the high burden of hypercholesterolemia. Aggressive cholesterol reduction protocols are advocated in this setting. Only few studies has examined the relationship between AVS and diabetes and they offered contradictories results then there is a high need to do a multicentric study to verify our findings in diabetic population.

\section{References}

${ }^{1}$ Rossi A, Targher G, Zoppini G, Cicoira M, Bonapace S, Negri C. Aortic and Mitral Annular Calcifications Are Predictive of AllCause and Cardiovascular Mortality in Patients With Type 2 Diabetes. Diabetes Care 2012; 35:1781-1786.

${ }^{2}$ Mantovani A, Pernigo M , Bergamini C , Bonapace S, Lipari P. Valbusad $\mathrm{F}$ et al. Heart valve calcification in patients with type 2 diabetes and nonalcoholic fatty liver disease. Metabolism and Clinical Experimental 2015; 64: 879-887.
Table I. Association between the presence of AVS and demographic variables, duration of diabetes and BMI

\begin{tabular}{|c|c|c|c|c|c|c|c|}
\hline \multirow[t]{2}{*}{ Item } & \multicolumn{2}{|c|}{$\begin{array}{c}\text { AVS } \\
(n=18)\end{array}$} & \multicolumn{2}{|c|}{$\begin{array}{l}\text { Without } \\
\text { AVS } \\
(n=61)\end{array}$} & \multicolumn{2}{|c|}{$\begin{array}{c}\text { Total } \\
(n=79)\end{array}$} & \multirow[t]{2}{*}{$\begin{array}{l}\text { Chi-square } \\
\text { statistic }\end{array}$} \\
\hline & No. & $\%$ & No. & $\%$ & No. & $\%$ & \\
\hline \multicolumn{8}{|c|}{ GENDER } \\
\hline Female & 8 & 44.4 & 22 & 36.1 & 30 & 38.0 & \multirow{2}{*}{$\begin{array}{c}X^{2}=0.1349 \\
P=0.35\end{array}$} \\
\hline Male & 10 & 55.6 & 39 & 63.9 & 49 & 62.0 & \\
\hline \multicolumn{8}{|c|}{ AGE GROUPS (YEARS) } \\
\hline $35-44$ & - & - & 13 & 21.3 & 13 & 16.5 & \multirow{4}{*}{$\begin{aligned} X^{2} & =15.430 \\
p & =0.001\end{aligned}$} \\
\hline $45-54$ & 5 & 27.7 & 23 & 37.7 & 28 & 35.4 & \\
\hline $55-64$ & 5 & 27.7 & 20 & 32.8 & 25 & 31.6 & \\
\hline $65-74$ & 8 & 44.4 & 5 & 8.2 & 13 & 16.5 & \\
\hline \multicolumn{8}{|c|}{ DURATION OF DIABETES (YEARS) } \\
\hline 0 a 4 & 2 & 11.1 & 23 & 37.7 & 25 & 31.6 & \multirow{3}{*}{$\begin{array}{c}X^{2}=4.965 \\
P=0.08\end{array}$} \\
\hline 5 a 9 & 7 & 38.8 & 20 & 32.8 & 27 & 34.2 & \\
\hline 10 a 30 & 9 & 50.0 & 18 & 29.5 & 27 & 34.2 & \\
\hline \multicolumn{8}{|c|}{ BODY MASS INDEX GROUPS } \\
\hline Normal & 3 & 16.6 & 5 & 8.2 & 8 & 10.1 & \multirow{3}{*}{ Nil } \\
\hline Overweight & II & 61.2 & 40 & 65.6 & 51 & 64.6 & \\
\hline Obesity & 4 & 22.2 & 16 & 26.2 & 20 & 25.3 & \\
\hline
\end{tabular}

Table 2. Association between the presence of AVS and the variables of metabolic control.

\begin{tabular}{|c|c|c|c|c|c|c|c|}
\hline \multirow{2}{*}{ Item } & \multicolumn{2}{|c|}{$\begin{array}{c}\text { AVS } \\
(n=18)\end{array}$} & \multicolumn{2}{|c|}{$\begin{array}{c}\text { Without } \\
\text { AVS } \\
(n=6 I)\end{array}$} & \multicolumn{2}{|c|}{$\begin{array}{c}\text { Total } \\
(n=79)\end{array}$} & \multirow{2}{*}{$\begin{array}{l}\text { Chi-square } \\
\text { statistic }\end{array}$} \\
\hline & No. & $\%$ & $\begin{array}{l}\mathbf{N} \\
\mathbf{o}\end{array}$ & $\%$ & $\begin{array}{l}\mathbf{N} \\
\mathbf{o .}\end{array}$ & $\%$ & \\
\hline \multicolumn{8}{|c|}{ TOTAL CHOLESTEROL } \\
\hline Normal & 6 & 33.3 & 36 & 59.1 & 42 & 53.2 & \multirow{2}{*}{$\begin{aligned} X^{2} & =2.723 \\
p & =0.04\end{aligned}$} \\
\hline Increase & 12 & 66.6 & 25 & 40.9 & 37 & 46.8 & \\
\hline \multicolumn{8}{|c|}{ TRYGLYCERIDES } \\
\hline Normal & 4 & 22.2 & 17 & 27.9 & 21 & 26.6 & \multirow{2}{*}{$\begin{aligned} X^{2} & =0.029 p \\
& =0.43\end{aligned}$} \\
\hline Increase & 14 & 77.8 & 44 & 72.1 & 58 & 73.4 & \\
\hline \multicolumn{8}{|c|}{ FASTING SERUM GLUCOSE } \\
\hline Hypoglycemia & -1 & - & 2 & 3.2 & 2 & 2.5 & \multirow{3}{*}{$\begin{aligned} X^{2} & =0.038 p \\
& =0.42\end{aligned}$} \\
\hline Adequate control & 7 & 38.8 & 18 & 29.5 & 25 & 31.6 & \\
\hline $\begin{array}{l}\text { Inadequate } \\
\text { control }\end{array}$ & II & 61.2 & 41 & 67.2 & 52 & 65.9 & \\
\hline \multicolumn{8}{|c|}{ POSTPRANDIAL SERUM GLUCOSE } \\
\hline Hypoglycemia & - & - & 1 & 1.6 & 1 & 1.3 & \multirow{3}{*}{$\begin{aligned} X^{2} & =0.379 P \\
& =0.26\end{aligned}$} \\
\hline Adequate control & 5 & 27.8 & 23 & 37.8 & 28 & 35.4 & \\
\hline $\begin{array}{c}\text { Inadequate } \\
\text { control }\end{array}$ & 13 & 72.2 & 37 & 60.6 & 50 & 63.3 & \\
\hline \multicolumn{8}{|c|}{ HbAIC (\%) } \\
\hline Adequate control & 4 & 22.2 & 10 & 16.4 & 14 & 17.7 & \multirow{2}{*}{$\begin{aligned} X^{2} & =0.047 p \\
& =0.41\end{aligned}$} \\
\hline $\begin{array}{l}\text { Inadequate } \\
\text { control }\end{array}$ & 14 & 77.8 & 51 & 83.6 & 65 & 82.3 & \\
\hline
\end{tabular}

